OPEN ACCESS

Edited by:

Liang Cheng,

Harbin Medical University, China

Reviewed by:

Nguyen Quoc Khanh Le,

Taipei Medical University, Taiwan

Jialiang Yang,

Geneis (Beijing) Co. Ltd., China

${ }^{*}$ Correspondence:

Lei Xu

csleixu@szpt.edu.cn

Lijun Zhang

c7zlj@szpt.edu.cn

Fusheng Yu

fushengyu1@163.com

Specialty section:

This article was submitted to Molecular and Cellular Pathology,

a section of the journa

Frontiers in Cell and Developmental

Biology

Received: 03 November 2021 Accepted: 06 December 2021

Published: 23 December 2021

Citation:

Chen Z, Guo Y, Zhao D, Zou Q, Yu F,

Zhang $L$ and $X u L$ (2021)

Comprehensive Analysis Revealed that CDKN2A is a Biomarker for Immune Infiltrates in Multiple Cancers. Front. Cell Dev. Biol. 9:808208. doi: 10.3389/fcell.2021.808208

\section{Comprehensive Analysis Revealed that CDKN2A is a Biomarker for Immune Infiltrates in Multiple Cancers}

\author{
Zheng Chen ${ }^{1,2}$, Yingjie Guo ${ }^{1,3}$, Da Zhao ${ }^{1,2}$, Quan Zou ${ }^{1}$, Fusheng Yu ${ }^{4 *}$, Lijun Zhang ${ }^{2 *}$ and \\ Lei $X u^{3 *}$ \\ ${ }^{1}$ Institute of Fundamental and Frontier Sciences, University of Electronic Science and Technology of China, Chengdu, China, \\ ${ }^{2}$ School of Applied Chemistry and Biological Technology, Shenzhen Polytechnic, Shenzhen, China, ${ }^{3}$ School of Electronic and \\ Communication Engineering, Shenzhen Polytechnic, Shenzhen, China, ${ }^{4}$ Beidahuang Industry Group General Hospital, Harbin, \\ China
}

The CDKN2A (cyclin dependent kinase inhibitor 2A/multiple tumor suppressor 1) gene, also known as the $P 16$ gene, encodes multiple tumor suppressor 1 (MTS1), which belongs to the INK4 family. In tumor tissue, CDKN2A has a high expression level compared with normal tissue and reflects prognosis in tumor patients. Our research targeted the analysis of CDKN2A expression in 33 tumors and clinical parameters, patient prognosis and tumor immunity roles. The CDKN2A expression level was significantly correlated with the tumor mutation burden (TMB) in 10 tumors, and the expression of CDKN2A was also correlated with MSI (microsatellite instability) in 10 tumors. CDKN2A expression was associated with infiltrating lymphocyte (TIL) levels in 22 pancancers, thus suggesting that CDKN2A expression is associated with tumor immunity. Enrichment analysis indicated that CDKN2A expression was involved in natural killer cell-mediated cytotoxicity pathways, antigen processing and presentation, olfactory transduction pathways, and regulation of the autophagy pathway in multiple cancers. CDKN2A was significantly associated with several immune cell infiltrates in pantumors. CDKN2A may serve as a promising prognostic biomarker and is associated with immune infiltrates across cancers.

Keywords: CDKN2A, TMB, MSI, immune infiltrates, pan-cancer

\section{INTRODUCTION}

In recent years, malignant tumor incidence has increased year by year, and finding effective treatment methods for malignant tumors has been a research focus worldwide (Cheng et al., 2018; Zhao et al., 2019). At present, the effect of tumor treatment is unsatisfactory, and tumorigenesis is associated with gene variations. Mutations in cell cycle-related genes usually lead to tumor formation, such as TP53, CDKN2A, RB1 and BRCA1.

The CDKN2A gene, also known as the P16 gene, encodes multiple tumor suppressor 1 (MTS1), which belongs to the INK4 family. The CDKN2A gene was identified by yeast two-hybrid protein correlation screening for proteins that interact with CDK4 (cyclin-dependent kinase 4) (Serrano et al., 1993). The CDKN2A gene, located on chromosome $9 \mathrm{p} 21$ and $8.5 \mathrm{~kb}$ in length, contains 3 exons and encodes a protein composed of 148 amino acids (Serra and Chetty, 2018). The P16 protein can bind to CDK4 and CDK6 (cyclin-dependent kinase 6) and inhibit the formation of kinase activity complexes by cyclin D (CD) and CDK4 (Yang et al., 1996). The kinase activity complex can prevent RB protein phosphorylation. Cells are stopped in the $\mathrm{G}$ phase by blocking the phosphorylation of RB protein and regulating the cell 
cycle. Mutation of the CDKN2A gene will remove the inhibition of the CyclinD-CDK4 complex. Then, $\mathrm{RB}$ protein phosphorylation results in abnormal cell cycle progression, and cells gain unlimited proliferation ability (Romagosa et al., 2011).

$C D K N 2 A$ tumor suppressors with mutations or gene loss are related to various tumors. Methylation of the P16 gene may be an important mechanism in the development of ovarian cancer. Compared with patients without P16 promoter methylation, ovarian cancer patients with $P 16$ promoter methylation have a significantly higher risk of disease progression (Todd et al., 2000; Cheng et al., 2016). However, promoter methylation of P16 genes cannot be used as a marker in early ovarian cancer diagnosis (Jiang et al., 2017). Melanoma is a cancer formed by the gradual accumulation of pathological mutations in normal melanocytes. Loss of the CDKN2A gene may participate in early invasion and metastasis of melanoma and suppress the initiation of invasion through inhibition of BRN2 in melanoma (Zeng et al., 2018). The most common gene mutations in human pancreatic cancer are Kras activation and P16 inactivation. According to research on human pancreatic cancer, the proto-oncogene Kras can induce the expression of P16, and blocking the induction of P16 by Kras can lead to tumor transformation and cancer cell metastasis (Chang et al., 2014). The CDKN2A methylation frequency is significantly higher in pancreatic cancer patients, which is related to patient survival (Tang et al., 2015). Such genetic events in the $C D K N 2 A$ gene may play an important role in pancreatic ductal carcinoma.

A homozygous deletion mutation of CDKN2A (P16) was found in 31 patients with 127 EGFR mutations in lung cancer treated with EGFR-TKIs. For patients with CDKN2A (P16) deletion mutations, the median progression-free survival was 5.3 and 10.5 months, respectively. Therefore, EGFR mutation with CDKN2A (P16) deletion mutation is associated with the development of lung cancer (Jiang et al., 2016). In a study of patients with lymphoma, $25 \%$ of CDKN2A (P16) deletion mutations and $22 \%$ of TP53 deletion mutations were detected in tumor tissues. Patients with deletion mutations in both CDKN2A and TP53 had an average survival of 1.8 years, significantly lower than 4.3 and 5.1 years for patients with deletion mutations in only one of the genes. Both CDKN2A and TP53 deletion mutations are closely associated with shortened overall survival (Delfau-Larue et al., 2015). Thus, CDKN2A deletion mutation is a poor prognostic indicator in mantle cell lymphoma. Studies on malignant glioma have shown that deletion mutations of $C D K N 2 A$ can be seen in $40.3 \%$ of cases, of which homozygous deletion accounts for $74 \%$, and homozygous deletion is more common in patients with primary malignant glioma (Cen et al., 2012; Hu et al., 2021a).

CDKN2A is a multiple tumor suppressor 1 (MTS1) which encoded a protein named P16. P16 can bind CDK4 and CDK6. P16 protein inhibits cyclin D (CD) and CDK4 to form a complex with kinase activity then regulates the cell cycle. Phase $\mathrm{G}$ and cell cycle are abnormal if the CDKN2A gene is mutated or deleted, and the cells obtain unlimited proliferation ability. Therefore, the study of CDKN2A gene is very important to further understand the impact of gene mutation on the development of cancer. Here, we sought to determine the CDKN2A gene deletion status in 33 kinds of tumors, and through analysis of the CDKN2A gene and patient survival, correlations between clinical tumor stage, tumor mutation load, microsatellite correlation, the relationships between tumor microenvironment, immune cell infiltration, gene expression and enrichment of GSEA, the relationship between the CDKN2A gene and tumor progression was assessed.

\section{DATA AND METHODS}

\section{Data Acquisition and Processing}

Thirty-three tumor transcription datasets, somatic mutation, and survival data were obtained from the UCSC Xena (http://xena. ucsc.edu/) database. There were 11,057 samples (10,327 tumor samples and 730 normal samples) for transcription data. The BiomaRt (version 2.44.4) package of $\mathrm{R}$ (version 4.0.2) was used for gene ID conversion. The dplyr (version 1.0.5) package of $\mathrm{R}$ (version 4.0.2) was used for CDKN2A gene extraction from the transcription data. To analyze the differential expression of the CDKN2A gene, the ggpubr (version 0.4.0) (https:/CRAN.Rproject.org/package=ggpubr) package was used in 33 tumors.

\section{Relevant Analysis of CDKN2A Expression and Prognosis of Tumor Patients}

Overall survival status information for tumor samples $(10,327)$ was downloaded from theUCSC Xena (http://xena.ucsc.edu/) database for 33 tumors. DSS (disease-specific survival), DFI (disease-free interval) and PFI (progression-free interval) status and time information were downloaded from TCGA Pan-Cancer (PANCAN) in the UCSC Xena (http://xena.ucsc. $\mathrm{edu} /$ ) database. The survival status and time information for the 33 tumors were extracted and used for prognostic analysis. According to the CDKN2A expression level, two groups with high and low expression levels were distinguished, and the prognostic value of $C D K N 2 A$ for the two groups in each tumor was analyzed using the OS (overall survival), DSS, DFI and PFI data. The CDKN2A risk ratio forest plot was plotted. The limma (version 3.44.3) (Ritchie et al., 2015), survival (version 3.210), survminer (version 0.4.9) and forestplot (version 1.10.1) $\mathrm{R}$ packages were used to perform this analysis.

\section{Analysis of CDKN2A Expression and TMB, Tumor Stage and MSI}

The tumor microenvironment contains two components, immune and stromal cells, which possess diagnostic and prognostic value in cancers (Hanahan and Weinberg, 2011; Fridman et al., 2012; Zhang et al., 2019; Hu et al., 2020; Islam et al., 2020; Hu et al., 2021b). Stage information was obtained from the UCSC Xena (http://xena.ucsc.edu/) database for all tumor samples, and we divided the stages into 4 . With all of the tumors, approximately 8099 samples were used for tumor stage analysis. The R packages limma (version 3.44.3) and ggpubr (version 0.4.0) were used for the analysis of tumor stages associated with CDKN2A expression. A total of 10,114 samples of 33 tumors for mutation data were used for tumor mutation 
burden (TMB) analysis. The Spearman correlation test and fmsb package (https://CRAN.R-project.org/package $=f m s b)$ of $\mathrm{R}$ were applied for analysis of the correlation between the CDKN2A gene and TMB. A total of 10,415 tumor samples were used to calculate the MSI score combined with the CDKN2A gene expression data for MSI analysis. The Spearman correlation test method and fmsb package were also applied to create a radar plot between the tumor and $C D K N 2 A$ genes.

\section{Analysis of the Tumor Microenvironment and CDKN2A Expression Levels}

The tumor microenvironment is mainly composed of stromal cells and immune cells, which play an important role in cancer prognosis. ESTIMATE is a tool for predicting tumor purity in tumor tissues (Yoshihara et al., 2013). Estimate and limma packages were used to estimate tumor purity by calculating the proportion of immune and stromal cells based on 11,057 samples from 33 tumors. The correlations of CDKN2A expression and the tumor microenvironment score were tested by the Spearman correlation test method. The $\mathrm{R}$ packages ggplot2 (version 3.3.3), ggpubr (version 0.4.0) and ggExtra (version 0.9 ) were applied to determine the correlation distribution.

\section{Correlation Analysis of Immune Cell Infiltration and CDKN2A Expression}

The 22 immune cell types found in tumors include naive B cells, naive CD4 T cells, memory $\mathrm{B}$ cells and resting memory CD4 $\mathrm{T}$ cells and so on (Newman et al., 2015). To estimate the infiltration of these 22 immune cell types, we used CIBERSORT (https://cibersort.stanford.edu/) to calculate their percentages based on RNAseq data (Newman et al., 2019). The limma (version 3.44.3) package was used for expression data processing, and CIBERSORT was used to compute these immune cell infiltration scores. Correlations between CDKN2A expression and the 22 immune cell infiltration scores were tested by the Spearman correlation test method. The $\mathrm{R}$ packages ggpubr (version 0.4.0), ggplot2 (version 3.3.3) and ggExtra (version 0 . 9) were applied to determine the correlation distribution.

\section{PPI Networks and Correlation of CDKN2A With Marker Genes}

The CDKN2A PPI network was constructed in the STRING database (https://www.string-db.org/) (Szklarczyk et al., 2019), which contains 31 coexpressed genes. The 31 coexpressed genes, 1 tumor-associated gene and 47 immune checkpoint genes from the literature were applied to calculate the correlation with CDKN2A in 33 tumors with the Spearman correlation test and limma (version 3.44.3) package. The correlation heatmap of CDKN2A and 79 genes was constructed by the reshape2 (version 1.4.4) and RColorBrewer (version 1.1-2) packages.

\section{Analysis of Gene Enrichment}

GSEA (genome set enrichment analysis) is a method to determine whether a set of genes shows concordant differences between two biological states. Thirty-three tumor expression datasets were separated according to the $C D K N 2 A$ expression level. The phenotype label was $C D K N 2 A$ expression level. Based on the KEGG database (https://www.kegg.jp/) (Kanehisa and Goto, 2000) and GO database, genes were enriched into clusters. In addition, normalized enrichment scores were applied to classify the enriched pathways (Subramanian et al., 2005). FDR $<0.05$ was used for the enriched standard. The R packages limma (version 3. 44.3), org.Hs.eg.db (version 3.11.4), enrichplot (version 1.8.1) and clusterProfiler (version 3.16.1) were applied for GSEA.

\section{RESULTS}

\section{The mRNA Expression Levels of CDKN2A in Different Types of Cancers}

To verify the differential expression of $C D K N 2 A$ in tumor tissues, the CDKN2A transcriptome data of multiple cancers and normal tissues were analyzed using the ggpubr (version 4.0.4) package of $\mathrm{R}$. This analysis revealed that the CDKN2A expression level was higher in various tumor tissues. Figure 1 shows the differential expression of CDKN2A in all 33 tumors and normal tissues. This analysis revealed that $C D K N 2 A$ expression was extremely significant in 15 tumors compared with their normal tissue, including $\mathrm{KICH}$ (Kidney Chromophobe), BRCA (Breast invasive carcinoma), HNSC (Head and Neck squamous cell carcinoma), CHOL (Cholangiocarcinoma), COAD (Colon adenocarcinoma), KIRC (Kidney renal clear cell carcinoma), PRAD (Prostate adenocarcinoma), KIRP (Kidney renal papillary cell carcinoma), THCA (Thyroid carcinoma), LIHC (Liver hepatocellular carcinoma), STAD (Stomach adenocarcinoma), LUAD (Lung adenocarcinoma), UCEC (Uterine Corpus Endometrial Carcinoma) and READ (Rectum adenocarcinoma). In addition, SARC (Sarcoma), GBM (Glioblastoma multiforme), BLCA (Bladder Urothelial Carcinoma), LUSC (Lung squamous cell carcinoma) and CESC (Cervical squamous cell carcinoma and) tumors were significantly different from normal tissues. No change in expression levels was observed in the remaining 14 tumors. Abnormal expression of CDKN2A may be related to the occurrence of many cancers.

\section{Prognostic Value of CDKN2A in Pan-Cancer}

The impact of CDKN2A on the prognostic value of tumor samples was analyzed and compared with that of normal samples. CDKN2A with high expression was associated with OS (overall survival) in 9 tumors, and CDKN2A also correlated with poor DSS (disease-specific survival) in 7 tumors (Figures 2A,B). KIRP, LIHC, PRAD and UCEC tumors had poorer DFI (disease-free interval) due to the high expression level of CDKN2A (Figure 2C). Additionally, high CDKN2A expression was positively related to poor PFI (progression-free interval) in 8 tumors (Figure 2D).

To further clarify the role of $C D K N 2 A$ in a single cancer, clinical data for single cancers were used for analysis. Similarly, high mRNA levels of CDKN2A correlated with overall survival (OS) in ACC (Adrenocortical carcinoma), KIRC, LIHC, MESO 

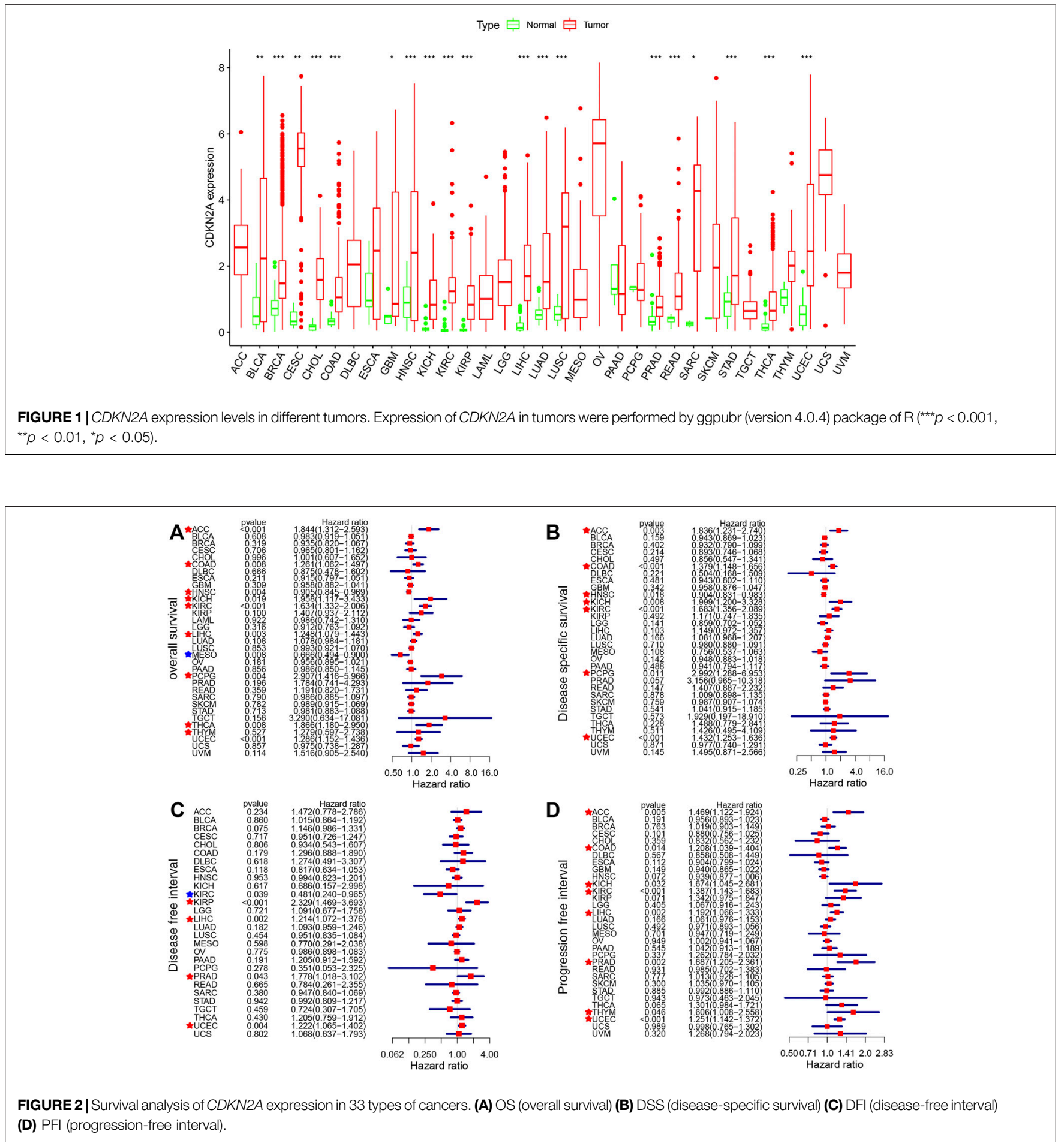

(Mesothelioma), PCPG (Pheochromocytoma and Paraganglioma) and UCEC tumors, with $p$-values of 0.010 , $0.029,0.001,0.001,0.005$ and 0.001 , respectively. For disease specific survival (DSS), high mRNA levels of CDKN2A correlated with ACC, COAD, DLBC (Lymphoid Neoplasm Diffuse Large B), KIRC, MESO, PCPG and UCEC tumors, with $p$-values of 0.007 ,
$0.017,0.049,0.006,0.001,0.015$, and 0.001 , respectively. The $p$-values of ACC, KIRC, LIHC, MESO, PARD, SKCM (Skin Cutaneous Melanoma) and UCEC tumors were 0.003, 0.028, $0.004,0.007,0.001,0.037$, and 0.001 , respectively, due to the high expression level of $C D K N 2 A$ in the progression-free interval (PFI). ESCA, KIRP and LIHC tumors were associated with 


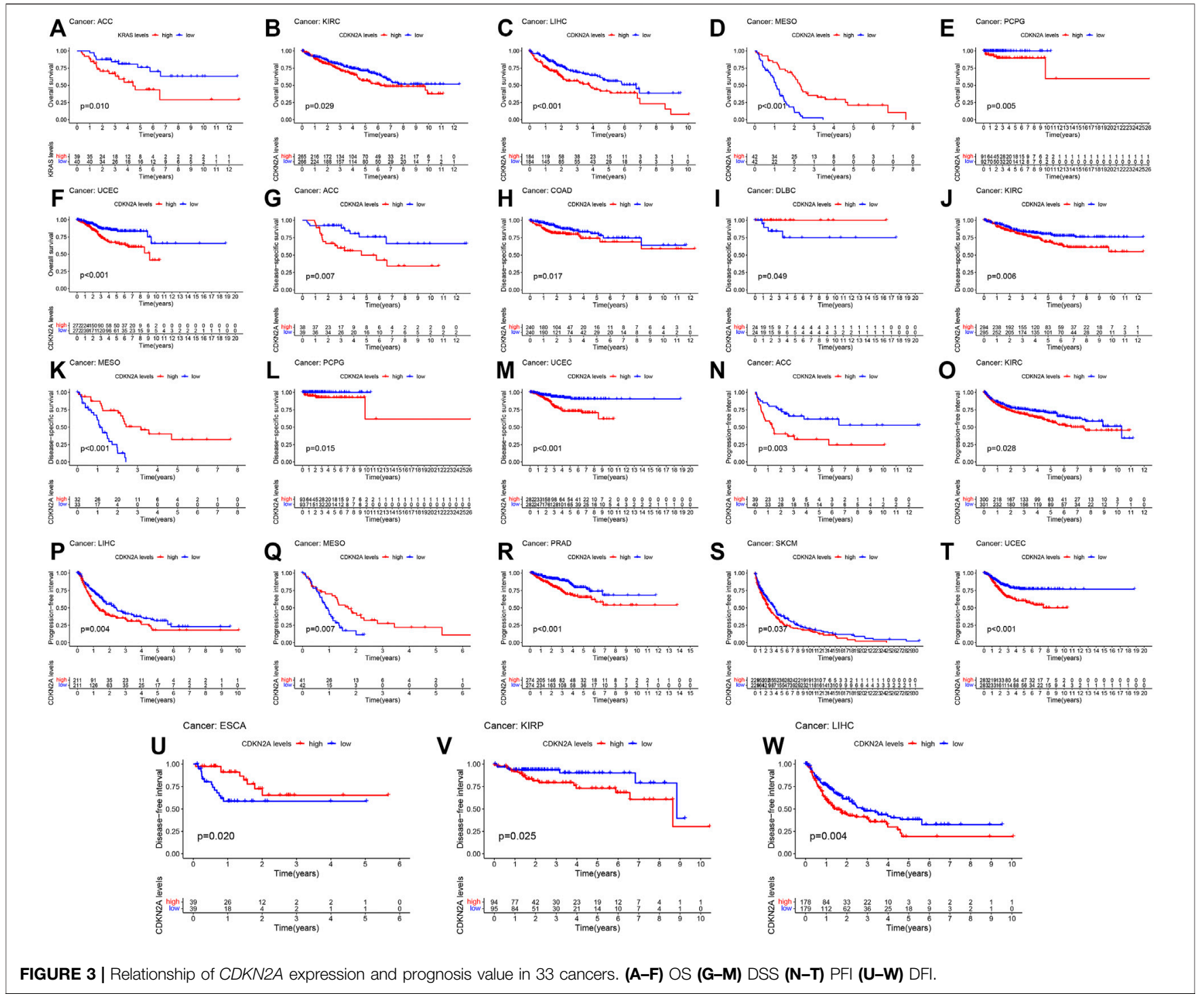

high expression of CDKN2A for the disease-free interval (DFI), with $p$-values of $0.020,0.025$ and 0.004 , respectively (Figure 3).

\section{CDKN2A Expression Levels Associated With the Clinicopathological Features of Patients With Tumors}

TMB (tumor mutation burden) and MSI (microsatellite instability) status are related to $C D K N 2 A$ expression for the clinicopathological characteristics of tumor stage pathology grade. To investigate this relationship, 33 tumor sample data points from the database were downloaded for analysis. Figures 4A-G shows that there was some effect on the high expression level of CDKN2A in seven tumors at several stages, specifically ACC, COAD, KICH, KIRC, KIRP, LIHC, and THCA. Moreover, CDKN2A with high expression levels in 10 tumors, BLCA, ACC, BRCA, THCA, PRAD, UCEC, LUAD, KICH, SKCM, KIRC, LIHC, and HNSC, was significantly correlated with TMB
(Figure 4H), and high expression levels of CDKN2A in BRCA, UCEC and PRAD tumors were also significantly correlated with microsatellite instability (MSI) (Figure 4I).

\section{CDKN2A Expression Levels Associated With Tumor Microenvironment}

For tumor microenvironment analysis, the estimate package of $\mathrm{R}$ was used to calculate the immune score and stromal score based on the CDKN2A expression level. Thirty-three tumor sample data points from the database were downloaded for analysis. As shown in Figure 5, the stromal score of LIHC and immune score of STAD were negatively correlated with CDKN2A expression levels. However, the immune scores of BRCA, CESC, KIRC, LGG (Brain Lower Grade Glioma), OV (Ovarian serous cystadenocarcinoma), READ, TGCT (Testicular Germ Cell Tumors), and THCA and the stromal scores of COAD, TGCT and THCA were negatively correlated with the CDKN2A 

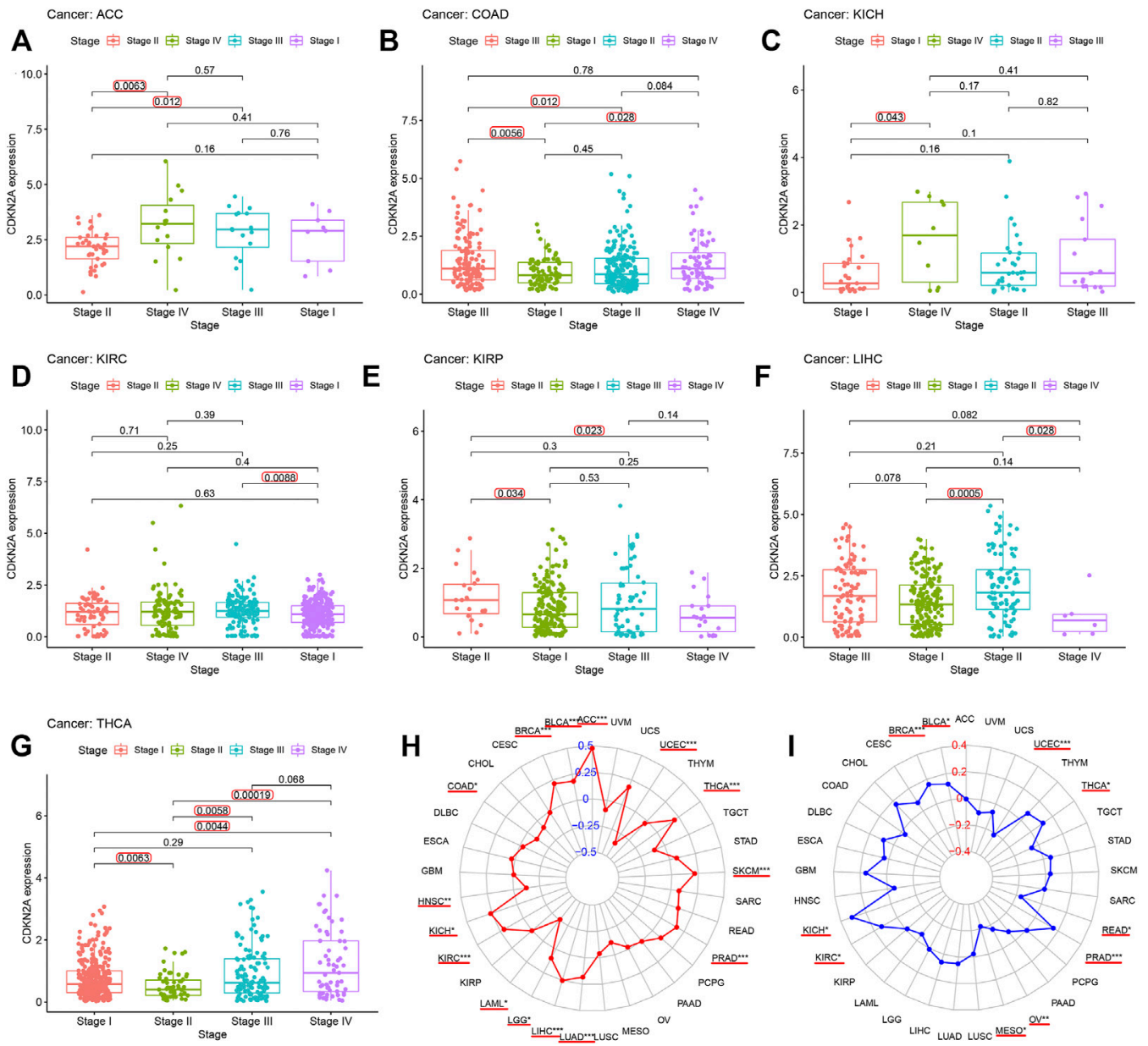

FIGURE 4 | Relationship between CDKN2A expression level and clinicopathological characteristics. (A-G) Relationship of CDKN2A expression level and stage grade. (H) Tumor mutation burden (TMB). (I) Microsatellite instability (MSI). ${ }^{*}, p<0.05 .{ }^{* \star} p<0.01,{ }^{* \star \star} p<0.001$.

expression level. The results showed that all immune and stromal scores of these tumors were significantly correlated with the CDKN2A expression level.

\section{The Association of CDKN2A With Tumor Immune Cell Infiltration Levels}

To investigate the relationship between immune cells and CDKN2A expression in tumors, 22 immune infiltrating cell expression datasets were prepared from the CIBERSORT website (https://cibersort.stanford.edu/runcibersort.php). High CDKN2A expression levels were positively associated with memory activated CD4 T cells, CD8 T cells, activated NK cells, regulatory $\mathrm{T}$ cells (Tregs), activated dendritic cells, and follicular helper $\mathrm{T}$ cells and were negatively related to the resting immune cell levels of mast cells, M2 macrophages, and memory resting CD4 $\mathrm{T}$ cells in BRCA. In HNSC, high CDKN2A expression was positively correlated with CD8 $\mathrm{T}$ cells and regulatory $\mathrm{T}$ cells (Tregs). High CDKN2A expression in KIRC was positively correlated with regulatory $\mathrm{T}$ cells (Tregs) and CD8 $\mathrm{T}$ cells and negatively correlated with $\mathrm{M} 2$ macrophages, resting memory CD4 T cells, and resting mast cells. CDKN2A expression in THCA tumors was positively associated with activated dendritic cells, regulatory $\mathrm{T}$ cells (Tregs), resting dendritic cells, activated memory CD4 memory $\mathrm{T}$ cells, and follicular helper $\mathrm{T}$ cells and negatively correlated with M0 macrophages and M2 macrophages. Furthermore, the results indicated that high expression of CDKN2A was negatively correlated with resting-state immune cells and positively correlated with the activation of immune cells in multiple tumors (Figure 6).

\section{Functional Analysis of CDKN2A}

The CDKN2A gene encodes multiple tumor suppressor 1, which regulates various cancers through different biological processes. The PPI network was used to investigate the molecular function of the CDKN2A protein using the STRING database (Figure 7A). The interacting genes from the protein-protein interaction network and common immune checkpoint genes were used to analyze the relationship with $C D K N 2 A$. The results indicated that several immune checkpoint genes and the genes from the PPI network were correlated with $C D K N 2 A$ expression levels in many tumors (Figure 7B). GSEA was used for the further analysis of 

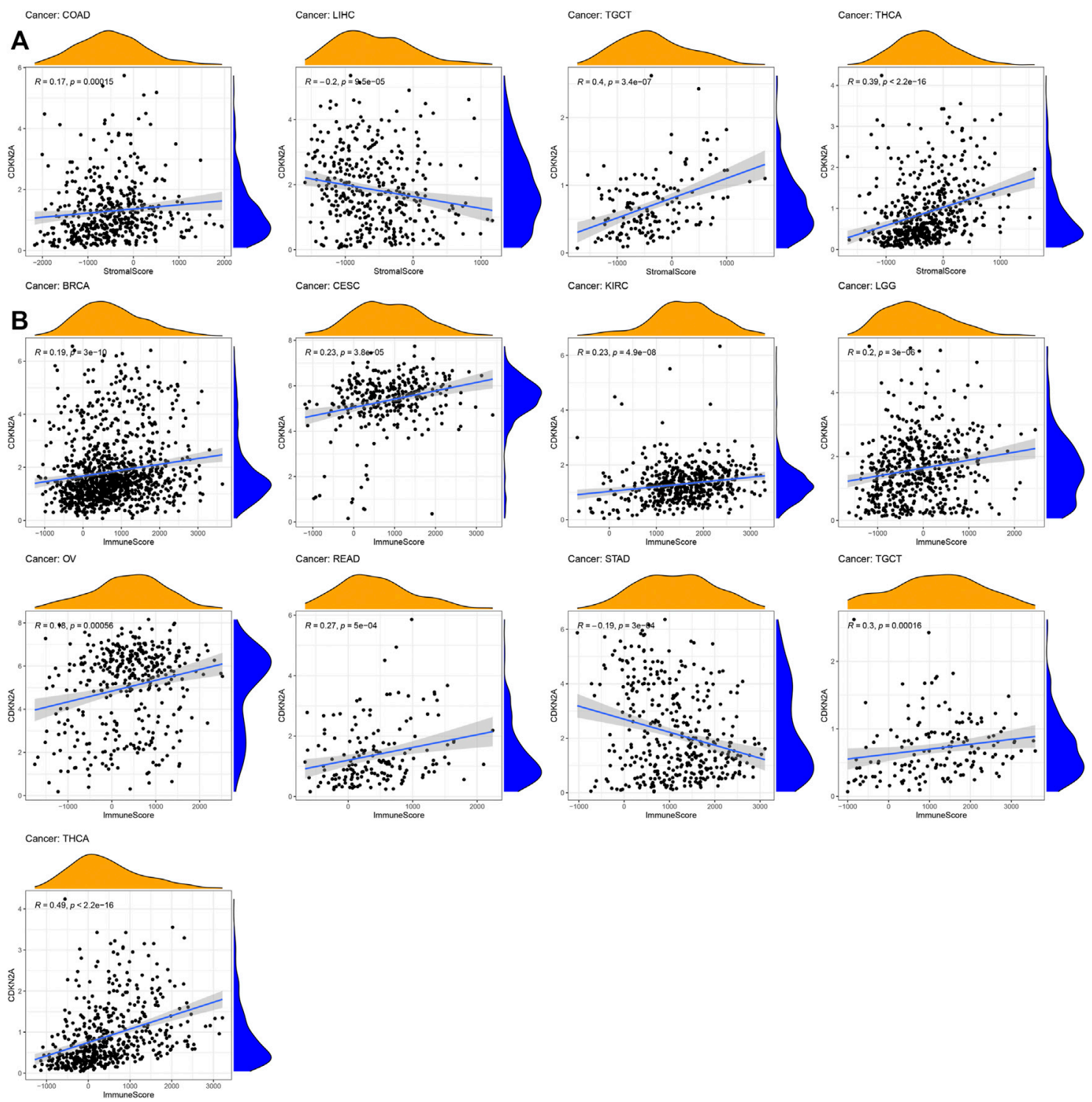

FIGURE 5 | Relationships between CDKN2A expression and tumor microenvironment. (A) Stromal score, (B) Immune score.

molecular mechanisms. High expression levels of $C D K N 2 A$ are involves in the immune response, regulation of signaling pathways and regulation of immune effector processes in ACC. In BLCA, CDKN2A promoted blood vessel endothelial cell migration and mRNA binding. In addition, a high expression level of CDKN2A participated in epidermal cell differentiation, epidermal development and the humoral immune response in BRCA, KIRC and OV tumors. The T cell receptor complex is involved in $\mathrm{KICH}$ and SKCM tumors due to high CDKN2A expression. High expression levels of $C D K N 2 A$ participated in keratinocyte differentiation and the intermediate filament cytoskeleton in LAML. High expression levels of CDKN2A participated in the immune response regulating signaling pathways and lymphocyte-mediated immunity intermediate filament cytoskeleton in LGG. In PCPGs, CDKN2A promoted
$B$ cell activation and the production of molecular mediators of the immune response. Circulating immunoglobulin complex and immune response regulating signaling pathways are involved in TGCT due to high CDKN2A expression (Figure 8A).

KEGG analysis suggested that a high CDKN2A expression level promoted many pathways in various tumors. In BRCA, CESE and OV, high CDKN2A expression levels are involved in antigen processing and presentation. High CDKN2A expression levels promoted natural killer cell-mediated cytotoxicity pathways in BRCA, OV and UVM (Uveal Melanoma). Olfactory transduction pathways are also involved in BRCA, OV, SARC, STAD, THCA, ESCA and LGG tumors. High CDKN2A expression levels promoted the regulation of the autophagy pathway in BRCA and CESC. In OV and UVM, 


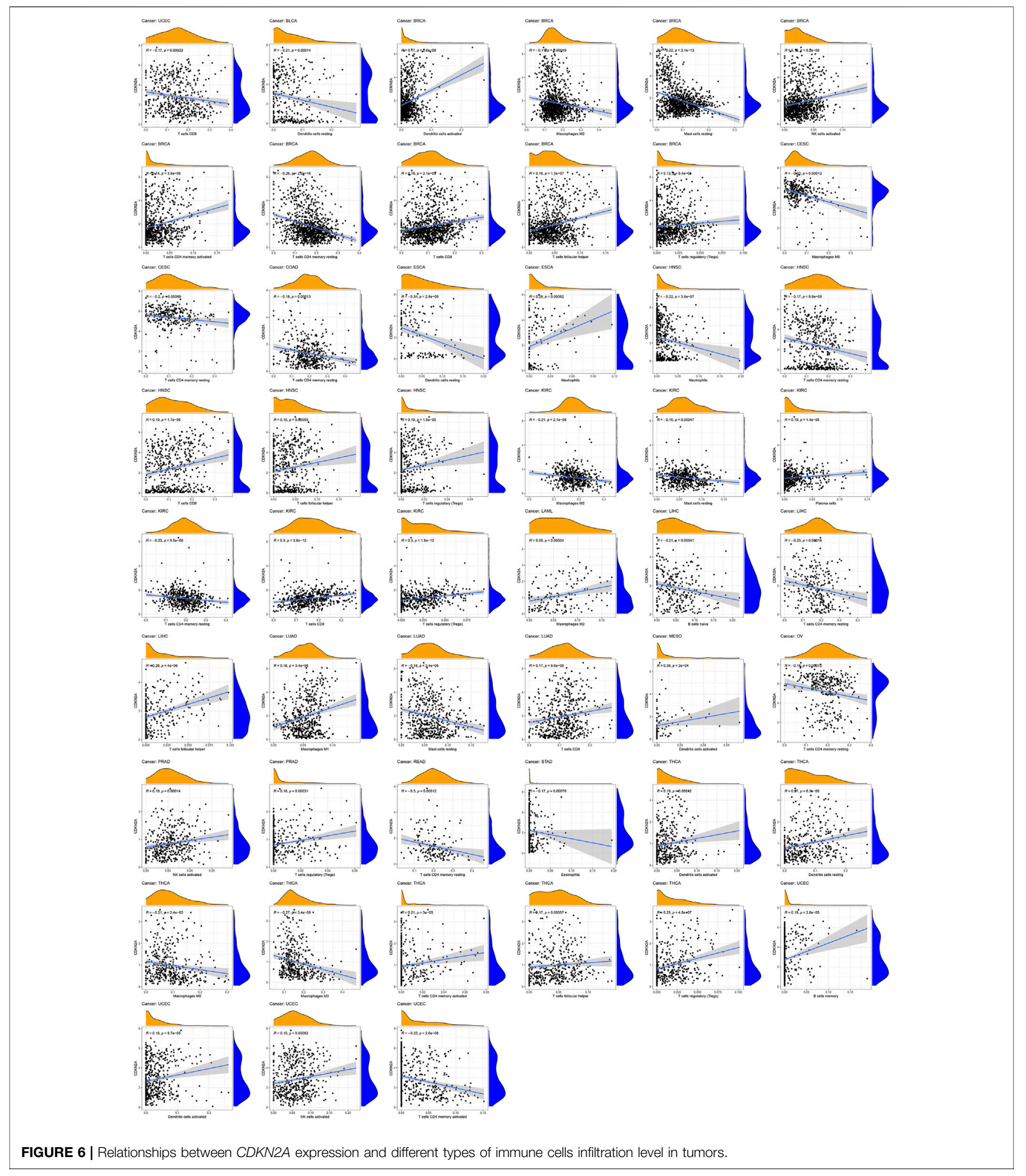



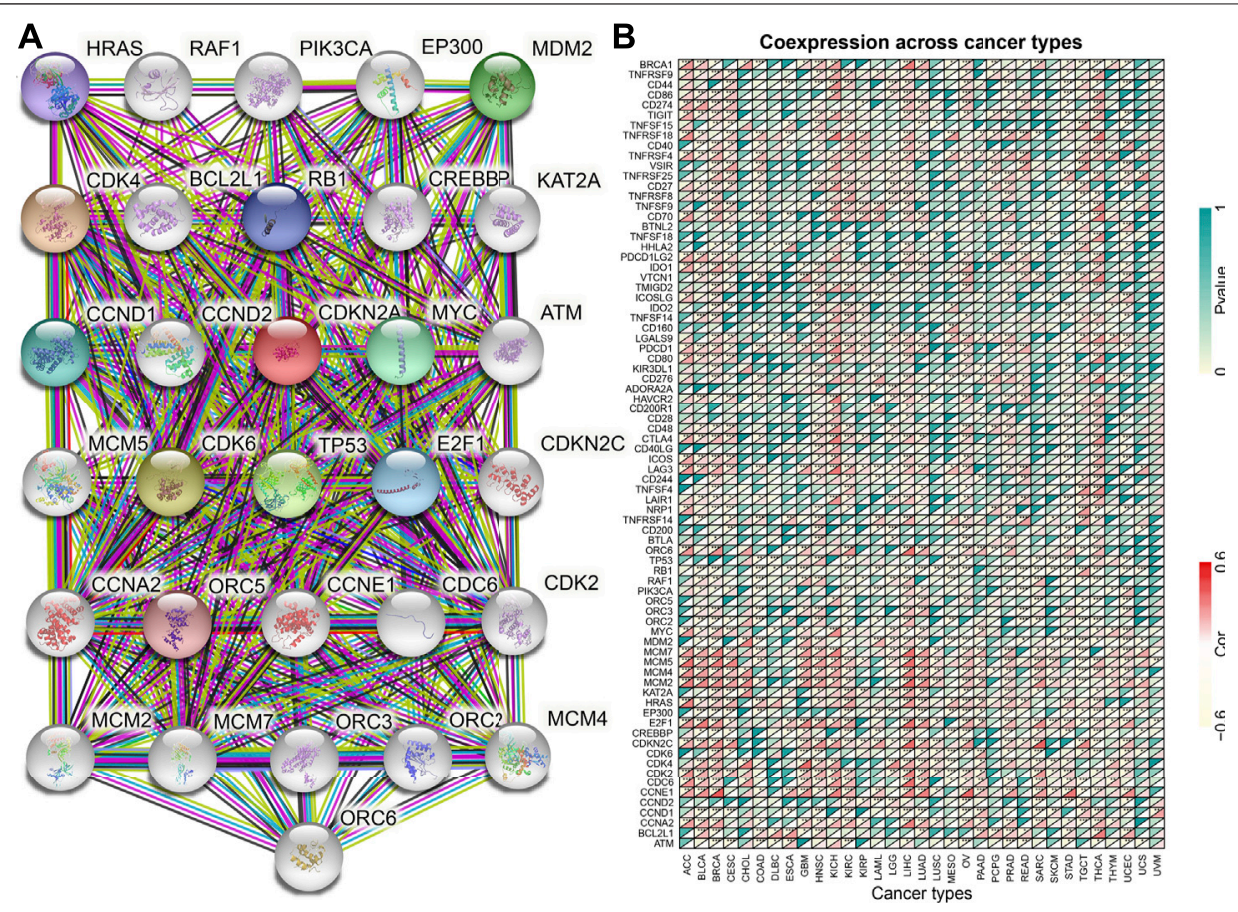

FIGURE 7|CDKN2A protein-protein network and expression relationships between CDKN2A and related genes. (A) PPI network for CDKN2A-interaction genes. (B) Correlation between CDKN2A expression and related genes (immune checkpoint genes and interacted related genes) expression.

high CDKN2A expression levels were involved in TOLL-like receptor signaling and the RIG-I-like receptor signaling pathway. CDKN2A has the potential to serve as a biomarker in various cancers (Figure 8B).

\section{DISCUSSION}

There are two proteins, p14ARF and p16INK4A, with tumor suppressive functions encoded by the CDKN2A locus (Sharpless and DePinho, 1999). The pl6INK4A protein is a CDK (cyclindependent kinase) inhibitor, and it functions as a predominant suppressor of melanoma (Krimpenfort et al., 2001; Sviderskaya et al., 2002; Goldstein et al., 2006; Bennett, 2015). The expression level of the CDKN2A gene was higher in tumor tissue than in normal tissue. Furthermore, CDKN2A expression was significantly higher in 15 tumors, which was consistent with tumor stage analysis in COAD, KIRC, KIRP, KIHC, LIHC and THCA. Several studies have indicated the prognostic role of CDKN2A in many tumors (Christopher et al., 2002; Ai et al., 2003; Zeng et al., 2018; Christodoulou et al., 2020; Ji et al., 2020; Xande et al., 2020). Therefore, CDKN2A plays an important prognostic role in these tumors.

Recently, tumor mutation burden (TMB) has been highlighted as a positive predictive factor and immunotherapy biomarker for immune checkpoint inhibitors and participates in immune checkpoint blockade therapy (Chan et al., 2018; Lee et al., 2019). MSI-high status is usually used to predict tumoragnostic markers and salvage immunotherapy in critically ill patients with end-stage cancer (Pietrantonio et al., 2020). The results indicated that TMB and high MSI were associated with high expression of $C D K N 2 A$ in a variety of tumors, such as BLCA, BRCA, UCEC, THCA, PRAD, KIRC and KICH. The relationship of $C D K N 2 A$ with MSI and TMB in this research is novel in these tumors, and CDKN2A is related to TMB and MSI and deserves more in-depth study.

Previous studies have shown that immune cells can recognize CDKN2A frameshift products (Hastings and Rausch, 2014). In mice, knockdown of $C D K N 2 A$ can reduce IL-4-induced IL-10 production, and IL-4 can induce IL-10-producing CD8(+) T cells (Zhao et al., 2013). CDKN2A gene occurs more frequently in cell lines than in pancreatic cancer tissues. Such genetic events on the CDKN2A gene may play an important role possibly at a later step in the progression of pancreatic ductal carcinoma (Sugimoto et al., 1998). Zeng et al. find that complete CDKN2A loss coincides with the onset of invasiveness in melanocytic tumors at distinct progression stages (Zeng et al., 2018). p16 inactivation was the major mechanism of RB pathway perturbation in nonsmall-cell lung carcinoma, with homozygous deletion being the most frequent method, followed by methylation and the rarer point mutations (Tam et al., 2013). The detection of homozygous CDKN2A deletion by FISH would have been helpful in confirming a diagnosis of mesothelioma over reactive mesothelial cells in 12 of 13 samples with positive or suspicious cytology (Illei et al., 2003). For tumor immune cell infiltration, the results suggested that $C D K N 2 A$ expression was positively related to CD8 $\mathrm{T}$ cells, NK activated cells and CD4 T activated cells but negatively related to memory resting CD4 T cells and resting CD4 
A

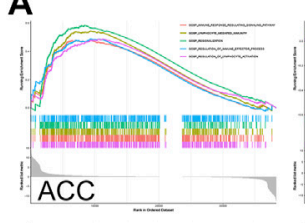

$\int_{\mid=1}^{\infty}$

DLBC
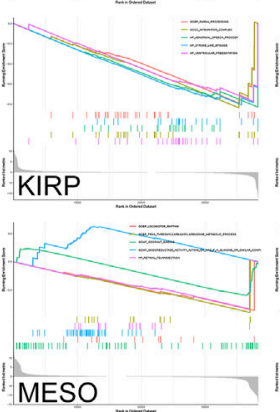

$\sqrt{2=}$

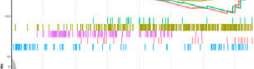

SARC

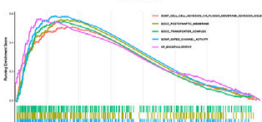

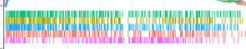

UCEC

B

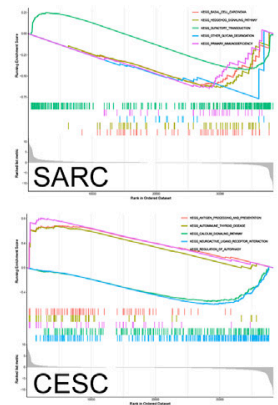

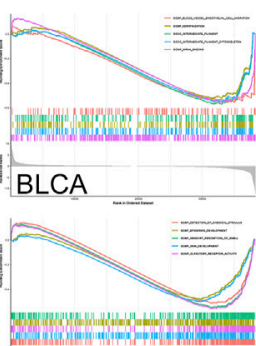
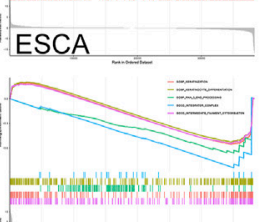

LAML
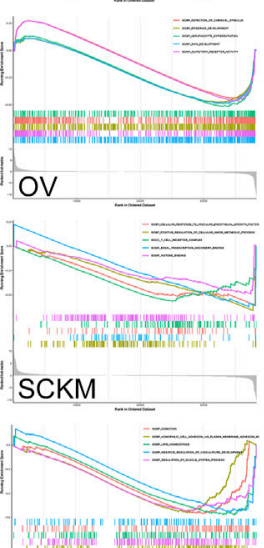

UCS
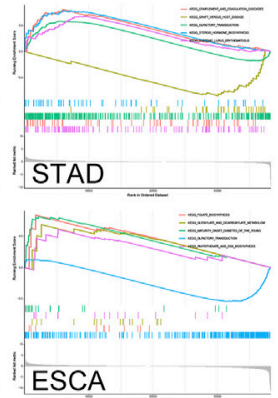
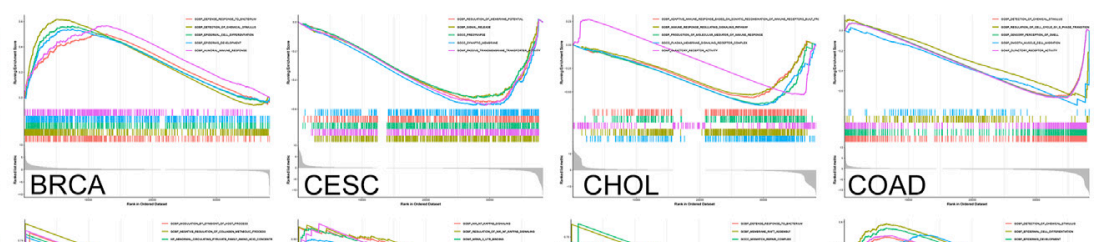

CESC
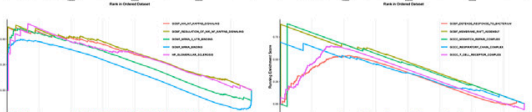

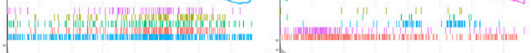

HNSC
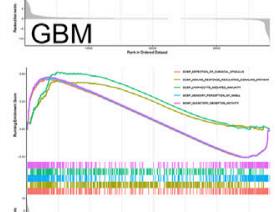

LGG
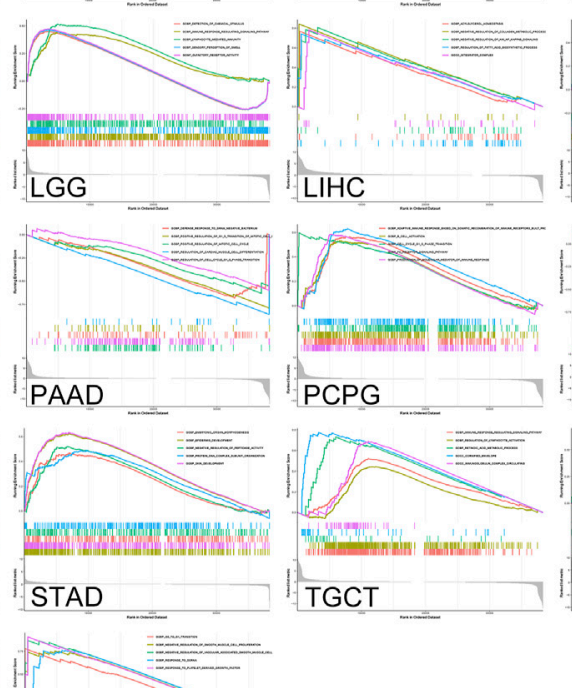

TGCT
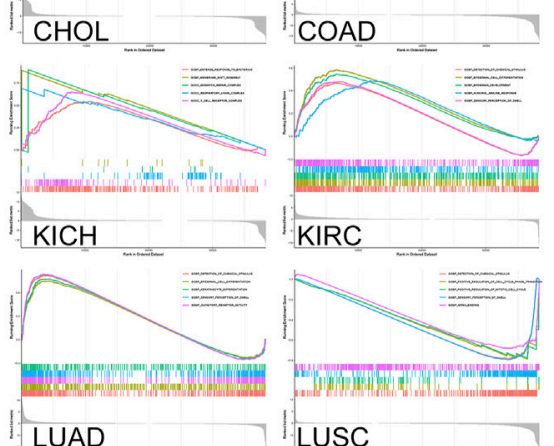

LUSC
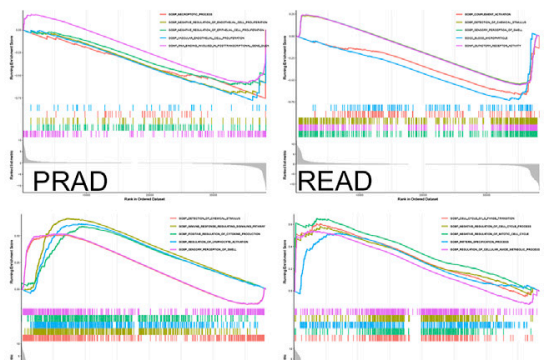

THCA

FIGURE 8 | GSEA enrichment analysis of CDKN2A expression in 33 tumors. (A) GO enrichment analysis in various tumors. (B) KEGG enrichment analysis in various tumors.

T cells. High expression of CDKN2A can increase activated immune cell numbers and reduce resting immune cell numbers. Our research showed that CDKN2A was associated with the infiltration of multiple immune cells in BRCA, HNSC, KIRC and THCA. In these tumors, $C D K N 2 A$ may be a potential immunotherapeutic biomarker to affect tumor cell viability.

The PPI network was analyzed in the STRING database, and 30 proteins interacted with CDKN2A, including CCND1, CDK1,
CCND2, CDK2, CDK6, CCNE1, CDK4, TP53, MYC, CDC6, and HRAS. Most of the interacting proteins belong to cell cycleassociated proteins and proto-oncogene-associated proteins. The apoptotic pathway contains 10 core genes (BAX, TP53, TP53INP1, CDKN2A, TP53BP1, CDKN1A, MDM2, CDKN1B, $C C D N 1$ and $B C L 2$ ), and these genes take part in the control of critical processes involved in hepatocellular carcinoma (HCC) (Yu et al., 2015). These genes may interact to control tumor 
progression. Disturbing the balance may result in a higher probability of tumor development (Cotter, 2009).

$C D K N 2 A$ as a biomarker can be applied to assess prognosis in cancers. In addition, $C D K N 2 A$ is expressed in various tumors and positively correlates with prognosis and immune cell infiltration, with high expression levels in most tumors. In general, the expression of CDKN2A played a detrimental role across cancers. In an analysis of immune cell infiltration, high CDKN2A expression was positively and significantly associated with many activated immune cells, which indicated that $C D K N 2 A$ may be involved in tumor immunity. Together, these integrated analyses suggested that $C D K N 2 A$ had prognostic value in cancers. Therefore, $C D K N 2 A$ has potential as a therapeutic target for tumor treatment. However, our study promotes future immunotherapy research.

\section{CONCLUSION}

Overall, we identified a novel cancer-related gene CDKN2A, which encodes multiple tumor suppressor 1 (MTS1) and belongs to the INK4 family. CDKN2A expression levels, clinical parameters, patient prognosis and tumor immunity were investigated in 33 tumors. In addition, we also explored the association between CDKN2A expression level and tumor mutation burden (TMB) and microsatellite instability (MSI). However, $C D K N 2 A$ expression is associated with infiltrating lymphocyte (TIL) levels, suggested that $C D K N 2 A$ expression is

\section{REFERENCES}

Ai, L., Stephenson, K. K., Ling, W., Zuo, C., Mukunyadzi, P., Suen, J. Y., et al. (2003). The P16 (CDKN2a/INK4a) Tumor-Suppressor Gene in Head and Neck Squamous Cell Carcinoma: A Promoter Methylation and Protein Expression Study in 100 Cases. Mod. Pathol. 16, 944-950. doi:10.1097/ 01.mp.0000085760.74313.dd

Bennett, D. (2015). Genetics of Melanoma Progression: The Rise and Fall of Cell Senescence. Pigment Cel Melanoma Res. 29, 122-140. doi:10.1111/pcmr.12422

Cen, L., Carlson, B. L., Schroeder, M. A., Ostrem, J. L., Kitange, G. J., Mladek, A. C., et al. (2012). p16-CDK4-RB axis Controls Sensitivity to a Cyclin-dependent Kinase Inhibitor PD0332991 in Glioblastoma Xenograft Cells. Neuro-oncology 14, 870-881. doi:10.1093/neuonc/nos114

Chan, T., Yarchoan, M., Jaffee, E., Swanton, C., Quezada, S. A., Stenzinger, A., et al. (2018). Development of Tumor Mutation Burden as an Immunotherapy Biomarker: Utility for the Oncology Clinic. Ann. Oncol. 30, 44. doi:10.1093/ annonc/mdy495

Chang, Z., Ju, H., Ling, J., Zhuang, Z., Li, Z., Wang, H., et al. (2014). Cooperativity of Oncogenic K-Ras and Downregulated p16/INK4A in Human Pancreatic Tumorigenesis. PloS one 9, e101452. doi:10.1371/journal.pone.0101452

Cheng, L., Hu, Y., Sun, J., Zhou, M., and Jiang, Q. (2018). DincRNA: a Comprehensive Web-Based Bioinformatics Toolkit for Exploring Disease Associations and ncRNA Function. Bioinformatics 34 (11), 1953-1956. doi:10.1093/bioinformatics/bty002

Cheng, L., Shi, H., Wang, Z., Hu, Y., Yang, H., Zhou, C., et al. (2016). IntNetLncSim: an Integrative Network Analysis Method to Infer Human lncRNA Functional Similarity. Oncotarget 7 (30), 47864-47874. doi:10.18632/oncotarget.10012

Christodoulou, E., Nell, R. J., Verdijk, R. M., Gruis, N. A., van der Velden, P. A., and van Doorn, R. (2020). Loss of Wild-type CDKN2A Is an Early Event in the related with tumor immunity. Based on these data, CDKN2A may be a promising prognostic biomarker with a potential molecular mechanism that affects survival outcomes in cancer patients.

\section{DATA AVAILABILITY STATEMENT}

The datasets presented in this study can be found in online repositories. The names of the repository/repositories and accession number(s) can be found in the article/supplementary material.

\section{AUTHOR CONTRIBUTIONS}

LX, LZ, FY, and QZ designed the research; ZC and DZ performed the research; ZC and YG analyzed the data; ZC wrote the manuscript. All authors read and approved the manuscript.

\section{FUNDING}

The work was supported by the National Natural Science Foundation of China (No.62002244, No.62002242, No.62001311) and Post-doctoral Foundation Project of Shenzhen Polytechnic (No. $6020330002 \mathrm{~K})$, the Research Foundation of Shenzhen Polytechnic (6021310019K). The funders had no role in study design, data collection and analysis, decision to publish, or preparation of the manuscript.

Development of Melanoma in FAMMM Syndrome. J. Invest. Dermatol. 140, 2298-e3. doi:10.1016/j.jid.2020.03.938

Christopher, S. A., Diegelman, P., Porter, C. W., and Kruger, W. D. (2002). Methylthioadenosine Phosphorylase, a Gene Frequently Codeleted with p16(cdkN2a/ARF), Acts as a Tumor Suppressor in a Breast Cancer Cell Line. Cancer Res. 62, 6639-6644. doi:10.1002/cncr.10960

Cotter, T. G. (2009). Apoptosis and Cancer: the Genesis of a Research Field. Nat. Rev. Cancer 9, 501-507. doi:10.1038/nrc2663

Delfau-Larue, M., Klapper, W., Berger, F., Jardin, F., Briere, J., Salles, G., et al. (2015). High-dose Cytarabine Does Not Overcome the Adverse Prognostic Value of CDKN2A and TP53 Deletions in Mantle Cell Lymphoma. Blood 126, 604-611. doi:10.1182/blood-2015-02-628792

Fridman, W. H., Pagès, F., Sautès-Fridman, C., and Galon, J. (2012). The Immune Contexture in Human Tumours: Impact on Clinical Outcome. Nat. Rev. Cancer 12, 298-306. doi:10.1038/nrc3245

Goldstein, A. M., Chan, M., Harland, M., Gillanders, E. M., Hayward, N. K., Avril, M.-F., et al. (2006). High-risk Melanoma Susceptibility Genes and Pancreatic Cancer, Neural System Tumors, and Uveal Melanoma across GenoMEL. Cancer Res. 66, 9818-9828. doi:10.1158/0008-5472.can-06-0494

Hanahan, D., and Weinberg, R. A. (2011). Hallmarks of Cancer: The Next Generation. Cell 144, 646-674. doi:10.1016/j.cell.2011.02.013

Hastings, K. T., and Rausch, M. P. (2014). Innate and Adaptive Immune Responses to Cancer. Fundamentals Cancer Prev., 81-121. doi:10.1007/978-3-64238983-2_4

$\mathrm{Hu}$, Y., Qiu, S., and Cheng, L. (2021). Integration of Multiple-Omics Data to Analyze the Population-specific Differences for Coronary Artery Disease. Comput. Math. Methods Med. 2021, 7036592. doi:10.1155/2021/7036592

Hu, Y., Sun, J-y., Zhang, Y., Zhang, H., Gao, S., Wang, T., et al. (2021). rs1990622 Variant Associates with Alzheimer's Disease and Regulates TMEM106B Expression in Human Brain Tissues. BMC Med. 19 (1), 11. doi:10.1186/ s12916-020-01883-5 
Hu, Y., Zhang, H., Liu, B., Gao, S., Wang, T., Han, Z., et al. (2020). rs34331204 Regulates TSPAN13 Expression and Contributes to Alzheimer's Disease with Sex Differences. Brain 143 (11), e95. doi:10.1093/brain/awaa302

Illei, P. B., Ladanyi, M., Rusch, V. W., and Zakowski, M. F. (2003). The Use of CDKN2A Deletion as a Diagnostic Marker for Malignant Mesothelioma in Body Cavity Effusions. Cancer 99 (1), 51-56. doi:10.1002/cncr.10923

Islam, M. A., Kundu, S., and Hassan, R. (2020). Gene Therapy Approaches in an Autoimmune Demyelinating Disease: Multiple Sclerosis. Curr. Gene Ther. 19 (6), 376-385. doi:10.2174/1566523220666200306092556

Ji, Z., Huo, C., and Yang, P. (2020). Genistein Inhibited the Proliferation of Kidney Cancer Cells via CDKN2a Hypomethylation: Role of Abnormal Apoptosis. Int. Urol. Nephrol. 52, 1049-1055. doi:10.1007/s11255-019-02372-2

Jiang, J., Gu, Y., Liu, J., Wu, R., Fu, L., Zhao, J., et al. (2016). Coexistence of p16/ CDKN2A Homozygous Deletions and Activating EGFR Mutations in Lung Adenocarcinoma Patients Signifies a Poor Response to EGFR-TKIs. Lung Cancer 102, 101-107. doi:10.1016/j.lungcan.2016.10.015

Jiang, Y., Yan, F., Liang, L., Wan, Y., Liu, J., and Cheng, W. (2017). Meta-analysis Demonstrates No Association between P16 Ink4a Promoter Methylation and Epithelial Ovarian Cancer. Arch. Gynecol. Obstet. 295, 697-704. doi:10.1007/ s00404-016-4264-x

Kanehisa, M., and Goto, S. (2000). KEGG: Kyoto Encyclopedia of Genes and Genomes. Nucleic Acids Res. 28, 27-30. doi:10.1093/nar/28.1.27

Krimpenfort, P., Quon, K. C., Mooi, W. J., Loonstra, A., and Berns, A. (2001). Loss of p16INK4a Confers Susceptibility to Metastatic Melanoma in Mice. Nature 413, 83-86. doi:10.1038/35092584

Lee, D.-W., Han, S. W., Bae, J. M., Jang, H., Han, H., Kim, H., et al. (2019). Tumor Mutation Burden and Prognosis in Colorectal Cancer Patients Treated with Adjuvant Fluoropyrimidine and Oxaliplatin. Clin. Cancer Res. 25, 6141. doi:10.1158/1078-0432.ccr-19-1105

Newman, A. M., Liu, C. L., Green, M. R., Gentles, A. J., Feng, W., Xu, Y., et al. (2015). Robust Enumeration of Cell Subsets from Tissue Expression Profiles. Nat. Methods 12, 453-457. doi:10.1038/nmeth.3337

Newman, A. M., Steen, C. B., Liu, C. L., Gentles, A. J., Chaudhuri, A. A., Scherer, F., et al. (2019). Determining Cell Type Abundance and Expression from Bulk Tissues with Digital Cytometry. Nat. Biotechnol. 37, 773-782. doi:10.1038/ s41587-019-0114-2

Pietrantonio, F., Loupakis, F., Randon, G., Raimondi, A., Salati, M., Trapani, D., et al. (2020). Efficacy and Safety of Immune Checkpoint Inhibitors in Patients with Microsatellite Instability-High End-Stage Cancers and Poor Performance Status Related to High Disease Burden. Oncologist 25, 803-809. doi:10.1634/ theoncologist.2020-0014

Ritchie, M. E., Phipson, B., Wu, D., Hu, Y., Law, C. W., Shi, W., et al. (2015). LIMMA powers Differential Expression Analyses for RNA-Sequencing and Microarray Studies. Nucleic Acids Res. 43, e47. doi:10.1093/nar/ gkv007

Romagosa, C., Simonetti, S., López-Vicente, L., Mazo, A., Lleonart, M. E., Castellvi, J., et al. (2011). p16Ink4a Overexpression in Cancer: a Tumor Suppressor Gene Associated with Senescence and High-Grade Tumors. Oncogene 30 (18), 2087-2097. doi:10.1038/onc.2010.614

Serra, S., and Chetty, R. (2018). p16. J. Clin. Pathol. 71 (10), 853-858. doi:10.1136/ jclinpath-2018-205216

Serrano, M., Hannon, G. J., and Beach, D. (1993). A New Regulatory Motif in CellCycle Control Causing Specific Inhibition of Cyclin D/CDK4. Nature 366 (6456), 704-707. doi:10.1038/366704a0

Sharpless, N. E., and DePinho, R. A. (1999). The INK4A/ARF Locus and its Two Gene Products. Curr. Opin. Genet. Dev. 9 (1), 22-30. doi:10.1016/s0959437x(99)80004-5

Subramanian, A., Tamayo, P., Mootha, V. K., Mukherjee, S., Ebert, B. L., Gillette, M. A., et al. (2005). Gene Set Enrichment Analysis: A Knowledge-Based Approach for Interpreting Genome-wide Expression Profiles. Proc. Natl. Acad. Sci. 102, 15545-15550. doi:10.1073/pnas.0506580102

Sugimoto, Y., Morita, R., Hikiji, K., Imura, G., Ogata, Y., Yasuda, D., et al. (1998). Alteration of the CDKN2A Gene in Pancreatic Cancers: Is it a Late Event in the
Progression of Pancreatic Cancer. Int. J. Oncol. 13, 669-676. doi:10.3892/ ijo.13.4.669

Sviderskaya, E. V., Simon, P. H., Tracy, J. E. -W., Lynda, C., Seth, J. O., David, J. E., et al. (2002). p16Ink4a in Melanocyte Senescence and Differentiation. J. Natl. Cancer Inst. 94, 446-454. doi:10.1093/jnci/94.6.446

Szklarczyk, D., Gable, A. L., Lyon, D., Junge, A., Wyder, S., Huerta-Cepas, J., et al. (2019). STRING V11: Protein-Protein Association Networks with Increased Coverage, Supporting Functional Discovery in Genome-wide Experimental Datasets. Nucleic Acids Res. 47 (D1), D607-d613. doi:10.1093/nar/gky1131

Tam, K. W., Zhang, W., Soh, J., Stastny, V., Chen, M., Sun, H., et al. (2013). CDKN2A/p16 Inactivation Mechanisms and Their Relationship to Smoke Exposure and Molecular Features in Non-small-cell Lung Cancer. J. Thorac. Oncol. 8 (11), 1378-1388. doi:10.1097/jto.0b013e3182a46c0c

Tang, B., Li, Y., Qi, G., Yuan, S., Wang, Z., Yu, S., et al. (2015). Clinicopathological Significance of CDKN2A Promoter Hypermethylation Frequency with Pancreatic Cancer. Sci. Rep. 5, 13563. doi:10.1038/srep13563

Todd, M. C., Sclafani, R. A., and Langan, T. A. (2000). Ovarian Cancer Cells that Coexpress Endogenous $\mathrm{Rb}$ and P16 Are Insensitive to Overexpression of Functional P16 Protein. Oncogene 19, 258-264. doi:10.1038/sj.onc.1203289

Xande, J. G., Dias, A. P., Tamura, R. E., Cruz, M. C., Brito, B., Ferreira, R. A., et al. (2020). Bicistronic Transfer of CDKN2A and P53 Culminates in Collaborative Killing of Human Lung Cancer Cells In Vitro and In Vivo. Gene Ther. 27, 51-61. doi:10.1038/s41434-019-0096-1

Yang, R., Serrano, M., Slater, J., Leung, E., and Koeffler, H. P. (1996). Analysis of p16INK4aand its Interaction with CDK4. Biochem. biophysical Res. Commun. 218, 254-259. doi:10.1006/bbrc.1996.0045

Yoshihara, K., Shahmoradgoli, M., Martínez, E., Vegesna, R., Kim, H., Torres-Garcia, W., et al. (2013). Inferring Tumour Purity and Stromal and Immune Cell Admixture from Expression Data. Nat. Commun. 4, 2612. doi:10.1038/ncomms3612

Yu, G. P., Xiao, Q. Y., Shi, Z. Q., Tang, L. S., Ma, X. P., Zhang, L. Y., et al. (2015). Genetic Polymorphisms in Apoptosis-Related Genes and the Prognosis of Hepatocellular Carcinoma. Am. J. Cancer Res. 5, 3249-3259.

Zeng, H., Jorapur, A., Shain, A. H., Lang, U. E., Torres, R., Zhang, Y., et al. (2018). Bi-allelic Loss of CDKN2A Initiates Melanoma Invasion via BRN2 Activation. Cancer cell 34, 56-68. e9. doi:10.1016/j.ccell.2018.05.014

Zhang, X., Shi, S., Shen, J., Zhao, M., and He, Q. (2019). Functional Immunoregulation by Heme Oxygenase 1 in Juvenile Autoimmune Diseases. Curr. Gene Ther. 19 (2), 110-116. doi:10.2174/ 1566523219666190710092935

Zhao, J., Sun, T., Wu, S., and Liu, Y. (2019). High Mobility Group Box 1: An Immune-Regulatory Protein. Curr. Gene Ther. 19 (2), 100-109. doi:10.2174/ 1566523219666190621111604

Zhao, Y., Zhao, H., Sun, Y., Hao, J., Qi, X., Zhou, X., et al. (2013). IL-4 Induces a Suppressive IL-10-producing CD8+ T Cell Population via a Cdkn2a-dependent Mechanism. J. Leukoc. Biol. 94, 1103-1112. doi:10.1189/jlb.0213064

Conflict of Interest: The authors declare that the research was conducted in the absence of any commercial or financial relationships that could be construed as a potential conflict of interest.

Publisher's Note: All claims expressed in this article are solely those of the authors and do not necessarily represent those of their affiliated organizations, or those of the publisher, the editors and the reviewers. Any product that may be evaluated in this article, or claim that may be made by its manufacturer, is not guaranteed or endorsed by the publisher.

Copyright (c) 2021 Chen, Guo, Zhao, Zou, Yu, Zhang and Xu. This is an open-access article distributed under the terms of the Creative Commons Attribution License (CC $B Y)$. The use, distribution or reproduction in other forums is permitted, provided the original author(s) and the copyright owner(s) are credited and that the original publication in this journal is cited, in accordance with accepted academic practice. No use, distribution or reproduction is permitted which does not comply with these terms. 


\section{GLOSSARY}

CDKN2A/MTS1 cyclin dependent kinase inhibitor 2A/multiple tumor suppressor 1

TMB tumor mutation burden

MSI microsatellite instability

TIL infiltrating lymphocyte

TP53 CDKN2A, RB1 and BRCA1.

CDK4 cyclin-dependent kinase 4

CDK6 cyclin-dependent kinase6

BRN2 POU class 3 homeobox 2

BAX BCL2 associated X, apoptosis regulator

TP53INP1 tumor protein p53 inducible nuclear protein 1

TP53BP1 tumor protein $\mathrm{p} 53$ binding protein 1

CDKN1A cyclin dependent kinase inhibitor 1A

MDM2 MDM2 proto-oncogene

CDKN1B cyclin dependent kinase inhibitor 1B

CCDN1 cyclin D1

BCL2 BCL2 apoptosis regulator

GSEA genome set enrichment analysis

UCSC Xena Xena Functional Genomics Explorer

OS overall survival

DSS disease-specific survival

DFI disease-free interval

PFI progression-free interval

PPI network protein-protein interact network

KEGG Kyoto Encyclopedia of Genes and Genomes

GO Gene Ontology

FDR false discovery rate

ACC Adrenocortical carcinoma

BLCA Bladder Urothelial Carcinoma

BRCA Breast invasive carcinoma

CESC Cervical squamous cell carcinoma and endocervical adenocarcinoma
CHOL Cholangiocarcinoma

COAD Colon adenocarcinoma

CDKN2A is a biomarker for multiple cancers

DLBC Lymphoid Neoplasm Diffuse Large B cell Lymphoma

ESCA Esophageal carcinoma

GBM Glioblastoma multiforme

HNSC Head and Neck squamous cell carcinoma

KICH Kidney Chromophobe

KIRC Kidney renal clear cell carcinoma

KIRP Kidney renal papillary cell carcinoma

LAML Acute Myeloid Leukemia

LGG Brain Lower Grade Glioma

LIHC Liver hepatocellular carcinoma

LUAD Lung adenocarcinoma

LUSC Lung squamous cell carcinoma

MESO Mesothelioma

OV Ovarian serous cystadenocarcinoma

PAAD Pancreatic adenocarcinoma

PCPG Pheochromocytoma and Paraganglioma

PRAD Prostate adenocarcinoma

READ Rectum adenocarcinoma

SARC Sarcoma

SKCM Skin Cutaneous Melanoma

STAD Stomach adenocarcinoma

TGCT Testicular Germ Cell Tumors

THCA Thyroid carcinoma

THYM Thymoma

UCEC Uterine Corpus Endometrial Carcinoma

UCS Uterine Carcinosarcoma

UVM Uveal Melanoma

IL-4 interleukin 4

IL-10 interleukin 10 